\title{
The use of visual and name codes in scanning and classifying colors
}

\author{
JOHN H. FLOWERS \\ University of Nebraska-Lincoln, Lincoln, Nebraska 68588 \\ and \\ SUSAN DUTCH \\ University of Connecticut, Storrs, Connecticut 06268
}

\begin{abstract}
The effect of incongruent color words on speed of classifying ink colors was measured in visual scanning tasks and in card sorting tasks. In both cases, little or no interference effects were noted when the classification allowed focusing on a single ink color or a set of highly similar colors (adjacent hues). Substantial interference occurred when the task required grouping of three dissimilar colors (nonadjacent hues). These findings suggest that the relative efficiency of name and visual codes in making perceptual classifications is largely dependent upon the memory requirements imposed by the task.
\end{abstract}

In a wide variety of experimental tasks, perceptual decisions may occur at any of several levels of processing. For example, comparisons of visually displayed letters can apparently occur on the basis of visual identity, name identity, or rule identity (Posner \& Mitchell, 1967). Similarly, Pisoni and Tash (1974) have suggested that comparisons of auditory phonemes can occur on the basis of a wave-form identity match or at a "higher" level of phonemic categorization. In these studies, different response speeds to identity and category matches seem to imply a hierarchical relationship between rapid sensory comparisons and "higher level" linguistic or conceptual comparisons. However, results of similar studies requiring sequential comparisons between stimuli (Cohen, 1969; Posner, Boies, Eichelman, \& Taylor, 1969; Tversky, 1969) suggest that visual and verbal stimulus information may be independently represented in parallel, and that either form of encoding may be used to make a perceptual decision. Subjects may thus be able to use the most efficient code for a perceptual decision. In simple matching tasks, this efficiency is determined by such factors as which type of representation is formed most quickly, has decayed the least with time, has been least disrupted by interpolated activity, or was "closest" to the type of stimulus the subject was expecting to match (Reed, 1973, Chapter 6).

Tasks such as speeded classification and memory scanning are more complex than simple perceptual matching in that they require storage of a set of percep-

This research was partially supported by the University of Nebraska Research Council, which included funding from Biomedical Grant RR7055-08 from NIH to the University of Nebraska. The authors would like to thank Gina Newbold, David Luby, and Beccy Blair for assistance in constructing materials and tabulating data. tual features or stimulus groupings for comparison with sensory input, of ten over a series of perceptual decisions. A much larger component of the response time is thus taken up by memory comparisons than in tasks requiring attention to only a single visual feature. Forming a name code from a visually displayed stimulus may take longer than forming a representation necessary for an image comparison, but owing to greater efficiency in scanning name codes (Weber \& Castleman, 1970), name comparisons may, with sufficient processing, lead to faster responses.

One behavioral consequence of a shift to name encoding in a visual classification task is that verbal stimulus dimensions influence response speed, whereas verbal attributes have little effect on tasks requiring only matching a single visual attribute (Egeth, Blecker, \& Kamlet, 1969). Flowers (1974) showed that printed trigrams could be sorted more rapidly into response categories which were correlated with a linguistic dimension than into categories with which the linguistic dimension varied orthogonally. For example, a task requiring the discrimination of "rat" and "hit" from "rit" and "dat" could be accomplished faster than the discrimination of "rit" and "hat" vs. "rat" and "dit," even though the correlation of individual letters with response categories is equivalent in both tasks. This facilitation effect of the correlated verbal dimension (meaningfulness, in the present example) was noted only for tasks for which stimulus assignment could not be made on the basis of a single letter; the sorting of "rat" and "hat" vs. "rit" and "dit" was no faster than for "rit" and "hit" vs. "rat" and "dat." These results strongly suggest the use of verbal encoding in tasks requiring the evaluation of several visual features, but the use of a rapid visual comparison or image match when only a single visual feature is critical. 
In addition to facilitation effects, verbal interference phenomena such as the Stroop effect (Stroop, 1935) provide a potential means for inferring the use of name vs. image codes in visual classification tasks. Our present experiments were directed at studying the use of image and name codes in classifying sets of ink colors by measuring the extent to which classification speed was reduced by the presence of competing verbal information. Specifically, we were interested in examining the relationship between the memory requirements of the task (in particular, the set size and visual encodability of the grouping of colors to be discriminated) and the amount of verbal interference which would be produced.

At a sensory or image level, color is important to both humans and many lower animals for locating, segregating, and identifying stimuli in the environment. Since scanning a list of color patches for instances of a single target color is not delayed when the patches form incongruent color names (Uleman \& Reeves, 1971), it would appear that visual search for colors involves "low-level" sensory comparisons which proceed more rapidly than name processing (Dyer, 1973; Egeth, Blecker, \& Kamlet, 1969). On the other hand, Hock and Egeth (1970) demonstrated considerable interference from incongruent color names in a classification task requiring yes-no judgments of whether ink colors belonged to a prespecified target set. The occurrence of Stroop-like interference in Hock and Egeth's task strongly suggests the use of name encoding in the decision process, which is susceptible to verbal response competition (Dalrymple-Alford \& Azkoul, 1972).

There are many nontrivial differences between simple scanning (Uleman \& Reeves, 1971) and the classification task used by Hock and Egeth (1970). In particular, the requirement of an overt verbal response in Hock and Egeth's task may have forced verbal interference which might not occur in a purely manual task. In addition, speeded classification and visual scanning may differ in the amount of visual information which must be stored prior to beginning the task. Perhaps a "visual" memory search is maximally efficient when subjects are required to store only a single color or a small group of highly similar colors for comparison with a series of stimuli, while a name search is more efficient when several visually dissimilar colors must be stored. If this were the case, one would expect to observe, in a purely manual classification task, an interaction between the Stroop effect and the number and sensory heterogeneity of the stimuli held in memory. In order to investigate these hypotheses, we chose two basic classification tasks commonly used to assess human performance: visual scanning and card sorting. In each type of task, subjects were required to make speeded discriminations based upon the ink color of stimuli (a) when colors were displayed as nonsense $\mathrm{X}$ patterns and (b) when the colors formed incongruent color names. In both the scanning and card sorting tasks, we varied both the number of different ink colors in response categories and the sensory similarity of colors within response categories. This provided us a means of assessing the combined effects of competing irrelevant verbal information, sensory organization factors, and "size" of the response categories per se, all within a set of tasks for which other factors (e.g., type of response required, method of displaying stimuli) remained relatively constant.

\section{EXPERIMENT I}

\section{Method}

Subjects. Twenty-four introductory psychology students each participated in a $1 \frac{1 / 2-h}{2}$ session, in partial fulfillment of course requirements. All subjects were right-handed, had normal color vision, and normal or corrected acuity.

Stimulus materials. Stimulus materials were lists of 125 color patches mounted in five columns of 25 stimulus patches each on $27.8 \times 21.5 \mathrm{~cm}$ white paper sheets. Since the task required subjects to mark through color patches by using a felt pen, each stimulus list was overlayed by a clear acetate sheet on each trial, allowing the stimulus lists to be reused. Each stimulus patch within a list was printed in one of seven ink colors: red, orange, yellow, green, blue, purple, or black. Two types of stimulus lists were used: $X$ lists, in which the color patches formed strings of three, four, five, or six Xs, and W lists, in which the colors formed incongruent color names (i.e., "Stroop" stimuli). Boldface capital letters approximately $.5 \mathrm{~cm}$ high were used.

Task and procedure. Subjects were required to scan through the columns of the stimulus lists, beginning with the leftmost column, marking with a felt pen each instance of an ink color belonging to a prespecified target set. One group of eight subjects scanned for instances of a single color (Set Size 1) on each trial. Four different colors were used as Set Size 1 targets: red (R), yellow (Y), purple (P), and blue (B). A second group of eight subjects searched for pairs of colors (Set Size 2) on each trial. These included red and orange (RO), red and green (RG), blue and purple (BP), and yellow and purple (YP). A third group of eight subjects searched for triplets of colors on each trial (Set Size 3). These included red, orange, and yellow (ROY); red, green, and blue (RGB); green, blue, and purple (GBP); and orange, green, and purple (OGP). The four Set Size 2 groupings and the four Set Size 3 groupings were selected to provide sets within which the targets were adjacent hues (e.g., GBP) and sets in which the colors were nonadjacent (e.g., OGP). The four Set Size 1 target colors were selected to provide a range of levels of visual discriminability from the nontarget colors in the list. In order to hold the number of positive responses constant across set size, different stimulus lists were constructed for each target set. Each list contained 51 positive instances of the color(s) belonging to the target set.'

Before beginning each experimental trial, the subject was shown a card containing the ink color(s) in the target set, displayed as $\mathrm{XXXX(s).} \mathrm{This} \mathrm{card} \mathrm{was} \mathrm{then} \mathrm{removed,} \mathrm{the} \mathrm{stimulus}$ sheet was placed in front of the subject and, on the oral signal of "Ready, set, go!," the subject began scanning the list. The subject orally signaled completion of scanning the list by saying "done." Scanning times were measured by a stopwatch. Errors were recorded by comparing the acetate sheet with a response key for each list.

Each subject received one practice and three experimental blocks of trials. Within each block, each of eight conditions (four target sets by two list types) was presented once. The order of conditions was determined by a Latin square, with each of the eight subjects in a set size group assigned to a row. These 
Table 1

Mean Search Time (in Seconds) and Error Rates for Target Sets of One Through Three Ink Colors in Experiment 1

\begin{tabular}{|c|c|c|c|c|c|c|c|c|}
\hline \multirow{4}{*}{ Set Size $1(\mathrm{SE}=.53)^{*}$} & \multicolumn{8}{|c|}{ Target Set } \\
\hline & \multicolumn{2}{|c|}{$\mathbf{R}$} & \multicolumn{2}{|c|}{$\mathrm{Y}$} & \multicolumn{2}{|c|}{ B } & \multicolumn{2}{|c|}{$\mathbf{P}$} \\
\hline & $\mathrm{x}$ & W & $\mathrm{X}$ & w & $\mathrm{X}$ & W & $\mathbf{X}$ & w \\
\hline & $\begin{array}{l}21.7 \\
(.29)\end{array}$ & $\begin{array}{r}20.7 \\
(.50)\end{array}$ & $\begin{array}{r}18.3 \\
(.04)\end{array}$ & $\begin{array}{l}18.5 \\
(.04)\end{array}$ & $\begin{array}{l}22.1 \\
(.33)\end{array}$ & $\begin{array}{r}21.0 \\
(.21)\end{array}$ & $\begin{array}{l}21.8 \\
(.21)\end{array}$ & $\begin{array}{r}20.5 \\
(.29)\end{array}$ \\
\hline \multirow[t]{3}{*}{ Set Size $2(S E=2.69)$} & \multicolumn{2}{|c|}{ RO } & \multicolumn{2}{|c|}{ RG } & \multicolumn{2}{|c|}{ BP } & \multicolumn{2}{|c|}{ YP } \\
\hline & $\mathrm{x}$ & $w$ & $\mathbf{x}$ & w & $\mathrm{x}$ & w & $\mathrm{x}$ & $w$ \\
\hline & $\begin{array}{l}32.7 \\
(.42)\end{array}$ & $\begin{array}{l}33.3 \\
(.42)\end{array}$ & $\begin{array}{l}38.5 \\
(.92)\end{array}$ & $\begin{array}{c}39.4 \\
(.96)\end{array}$ & $\begin{array}{l}34.0 \\
(.54)\end{array}$ & $\begin{array}{r}34.8 \\
(.50)\end{array}$ & $\begin{array}{c}36.9 \\
(1.46)\end{array}$ & $\begin{array}{r}36.9 \\
(1.04)\end{array}$ \\
\hline \multirow[t]{3}{*}{ Set Size $3(\mathrm{SE}=3.12)$} & \multicolumn{2}{|c|}{ ROY } & \multicolumn{2}{|c|}{ RYB } & \multicolumn{2}{|c|}{ GBP } & \multicolumn{2}{|c|}{ OGP } \\
\hline & $\mathrm{x}$ & $w$ & $\mathrm{x}$ & w & $\mathrm{x}$ & w & $\mathrm{x}$ & w \\
\hline & $\begin{array}{c}38.9 \\
(.17)\end{array}$ & $\begin{array}{l}40.0 \\
(.25)\end{array}$ & $\begin{array}{c}49.8 \\
(1.08)\end{array}$ & $\begin{array}{c}56.3 \\
(1.71)\end{array}$ & $\begin{array}{l}45.6 \\
(.62)\end{array}$ & $\begin{array}{l}46.3 \\
(.70)\end{array}$ & $\begin{array}{c}55.4 \\
(1.71)\end{array}$ & $\begin{array}{c}65.1 \\
(2.83)\end{array}$ \\
\hline
\end{tabular}

Note-Main cell entries are mean search times through lists of 125 stimuli; parentheses show mean number of errors per list of 125 stimuli. $\quad$ Standard error of the difference in times for list type (Xv. W), calculated from the subjects by list type mean square.

eight conditions resulted from the combination of the four target sets by two list types.

\section{Results}

Mean scanning times in seconds (across the three blocks of trials) for each condition are shown in Table 1 . Error rates (mean number of errors per list) are also shown in this table. However, as in most scanning and classification tasks, errors were both infrequent and correlated with scanning times. The scanning times were therefore used exclusively as the dependent variable in subsequent data analysis.

Set Size 1. Analysis of variance indicated a significant effect of the ink color $[F(3,21)=21.5, p<.001]$, and it appears from Table 1 that the preponderance of this effect rests in the faster scanning for yellow ink. A significant decrease in scanning times over blocks was also noted $[F(2,14)=5.6, p<.05]$, but there were no significant interactions between blocks and other variables. Thus, the overall scanning times for these single colors seem to be sensitive to changes in practice and visual discriminability. However, since the $\mathrm{X}$ lists actually produced slightly longer mean sorting times for three of the color sets, the data provide no evidence for a verbal interference (i.e., Stroop) effect. These findings essentially replicate those of Uleman and Reeves (1971), and support the theory that visual scanning for a single visual attribute can be accomplished independently of verbal mediation.

Set Size 2. For the Set Size 2 conditions, scanning times also differed as a function of target set $[F(3,21)=$ $7.5, \mathrm{p}<.01]$. No significant effect of list type was observed $[F(1,7)<1]$, indicating subjects could search about equally well through $\mathrm{X}$ and $\mathrm{W}$ lists. Thus, while overall scanning times were somewhat longer, these data reflect essentially the same pattern of results as those obtained with Set Size 1: differences attributable to sensory properties of the target stimuli, but no interference from competing color names.

Set Size 3. A very different and interesting pattern of results occurred for the tasks requiring scanning for three colors simultaneously. Not only did the target sets produce widely differing scanning times $[F(3,21)=$ $48.0, p<.005]$, but there was also a substantial verbal interference effect $[F(1,7)=28.6, p<.005]$. However, there was a Color Set by List Type interaction $[F(3,21)=10.4, p<.005]$, reflecting the much greater verbal interference with Sets RYB and OGP (approximately $13 \%$ and $18 \%$ increases in scanning times, respectively) than with Sets ROY and GBP (approximately $2 \%$ and $3 \%$ increases). In fact, the $2 \%$ longer times for GBP.W than for GBP-X resulted nearly exclusively from the data contributed by a single subject. It thus appears that verbal interference can be substantially avoided when scanning for three adjacent hues, even though substantial interference occurs when searching for three nonadjacent hues.

\section{Discussion}

Target set size, visual discriminability, and sensory heterogeneity all appear to influence the speed of visual scanning for a set of colors. The effects of the sensory factors on the scanning rates for the $X$ lists, together with the lack of substantial verbal interference for all but the three nonadjacent hue sets (RYB and OGP), strongly suggest visual rather than name encoding of the target colors. Comparisons between stored visual codes and the ink colors on the stimulus list would effectively constitute a visual "within analyzer" comparison (Treisman \& Fearnley, 1969) that is totally independent 
of verbal processing. The appearance of a rather large Stroop-like interference effect when subjects were required to search for three dissimilar colors, on the other hand, suggests the use of name-level processing. Whether the interference is the direct result of the adoption of a name encoding strategy forced by an excessive visual memory load or the indirect result of slowing down the visual processing time sufficiently that "automatic" verbal processing takes over, these findings demonstrate that the memory load is a critical factor in determining the level of processing at which the classification occurs. It would appear from the overall pattern of data that what constitutes this memory load is not, however, exclusively a function of set size, but is highly dependent on sensory organization factors as well.

\section{EXPERIMENT II}

Speeded classification is similar to visual scanning in that subjects must make speeded discriminations of sequentially displayed stimuli according to a classification scheme defined by the experimenter. Speeded classification may, however, require more than a binary stimulus classification, and always requires a motor response to each stimulus (rather than to only "positive" items). Additionally, the average time per stimulus is generally less in scanning than for speeded classification as measured by card sorting, although this is largely dependent upon the classification required (e.g., scanning times per stimulus ranged from $.146 \mathrm{sec}$ to $.521 \mathrm{sec}$ in Experiment I). Either the distinction between positive-negative and neutral response assignments (Gottwald \& Garner, 1972) or the classification time per stimulus could have a bearing upon the level of processing at which perceptual decisions occur. A comparison between the pattern of data obtained in Experiment $I$ and data obtained from a card sorting task using similar stimulus materials thus provides a means of evaluating the generality of circumstances in which verbal interference may occur in a "visual" task, as well as an opportunity to evaluate possible differences in the processing demands imposed by these two techniques.

\footnotetext{
Method

Stimulus materials. Each stimulus consisted of a color patch shaped as an incongruent color name or as an XXXX mounted on a white card. The size and lettering of color patches was identical to those used in the scanning lists in Experiment $I$. Each card was $8.9 \times 6.3 \mathrm{~cm}$, with the longer side vertical and a small piece cut off the upper left corner to maintain proper orientation. On a single trial, subjects were required to sort a deck of 30 or 36 such cards, according to the classification scheme required by the experimental conditions described below.

1:3 mappings. One set of four conditions required the sorting of decks of 36 cards containing red, yellow, green, and blue ink patches. These four conditions resulted from the combination of the two stimulus types (incongruent words or
}

XXXXs) with two target colors (yellow or blue). In Condition $\mathrm{Y}-\mathrm{X}$, subjects were required to separate 18 cards containing yellow XXXXs from cards containing blue, red, or green XXXXs (six instances of each). Similarly, Condition B-X required separating 18 cards containing blue XXXXs from cards containing red, yellow, or green XXXXs. In Conditions Y-W and $B-W$, the required groupings of ink colors were identical to $\mathrm{Y}-\mathrm{X}$ and $\mathrm{B}-\mathrm{X}$, respectively, but the colors were displayed as incongruent words. ${ }^{2}$ Since these conditions presumably allowed focusing upon a single color (Gottwald \& Garner, 1972), they are somewhat analogous to the Set Size 1 scanning tasks of Experiment $\mathrm{I}$.

2:2 mappings. Another set of four conditions required the sorting of decks of 36 cards containing nine instances each of red, blue, orange, and purple patches into two piles of 18 cards each. Four conditions resulted from the combination of two stimulus types ( $X$ and $W$ ) and two color sets ( $R O$ and $R B$ ). In Conditions RO-X and RO-W, the red and orange colors were sorted into one pile and the blue and purple colors into the other. Conditions $\mathrm{RB}-\mathrm{X}$ and $\mathrm{RB}-\mathrm{W}$ required a red-blue vs. orangepurple split.

3:3 mappings. A third set of four conditions required subjects to sort decks of 30 cards containing five instances each of the colors red, orange, yellow, green, blue, and purple. Conditions ROY-X and ROY-W required a red, orange, and yellow vs. green, blue, and purple split. Conditions RYB-X and RYB-W required a red, yellow, and blue vs. orange, green, and purple split.

Subjects. Students from an introductory psychology course volunteered as a means of fulfilling a course requirement. All subjects had normal color vision and normal or correctable acuity. Twelve subjects were assigned to the $1: 3$ mapping tasks, eight subjects to the $2: 2$ mapping tasks, and eight subjects to the $3: 3$ mapping tasks. ${ }^{3}$ Subjects were run individually in a session lasting about $1 \mathrm{~h}$.

Procedure. Before beginning the experiment, each subject was seated at a table and shown examples of the cards. He/she was then instructed thai all tasks would require sorting the cards on the basis of the ink color of the stimuli only, and not on the configuration or spelling of the ink patches. Prior to beginning each trial, the stimulus deck was shuffled and a card containing examples of the ink colors which were to be sorted into each category was shown to the subject. This card was removed from view when the subject indicated he/she understood the required classification." On each trial, subjects held the deck face up in one hand and, on the oral signal of "Ready, set, go!," dealt each card into the required pile "as rapidly as possible, avoiding errors." Sorting times were measured with a stopwatch; both time and errors were recorded following each trial. Subjects were informed about errors but not about times.

The subjects who worked the four $1: 3$ mapping conditions were given one "practice" and eight experimental blocks of trials in which each condition was given once. Data from practice blocks were not included in analysis. The subjects who worked the $2: 2$ conditions and the $3: 3$ conditions were given one practice and eight experimental blocks of trials within which each condition was given once. Order of conditions within blocks was determined by a Latin square.

\section{Results}

Error rates were extremely low; several subjects exhibited errorless performance, and the average error rate per deck across conditions was .20 for the 36 -card deck and .18 for the 30 -card decks. No detailed analysis of errors was therefore carried out. Since the $3: 3$ mappings required sorting decks of 30 cards, while the remainder of the decks required sorting 36 cards, the mean classification times per stimulus for each condi- 
tion, averaged across subjects and blocks of trials, are displayed in Table 2.

1:3 mappings. It is apparent from observation of Table 2, in which the difference among all four means is less than $10 \mathrm{msec} / \mathrm{card}$, that no significant or important verbal interference occurred. ${ }^{5}$ This pattern of results is thus similar to that observed in the singlecolor scanning tasks in Experiment I, suggesting rapid visual stimulus comparisons independent of verbal processing.

2:2 mappings. A comparison between the mean sorting times of RO-X and RO-W do not reveal any statistically significant interference effects $[t(7)=1.0$; plus RO-W produced longer mean sorting times for only five of the eight subjects]. On the other hand, the 31 - $\mathrm{msec} / \mathrm{card}$ difference $[\mathrm{t}(7)=2.89, \mathrm{p}<.05]$ between the means for RB-X and RB-W does appear to reflect some verbal interference; RB-W produced greater sorting times for seven of eight subjects and on six of seven blocks of trials. This pattern of results differs somewhat from that of the two-color scanning task obtained in the first experiment, in which no verbal interference was noted. One should be cautious, however, in attributing this discrepancy exclusively to differences between sorting and scanning, since the required groupings of stimuli within response categories (either red and blue or orange and purple) differed from those used in the target sets in the scanning task. Our data from the Set Size 3 conditions in Experiment I suggest that the sensory encodability of the color groupings may be as important as "set size" within response categories in determining whether verbal interference will result.

3:3 mappings. The mean sorting times of $3: 3$ mapping conditions displayed in Table 2 reveal influences of both the color classification itself (all eight subjects had shorter mean classification times for Condition ROY-X than for RYB-X), and a substantial verbal interference effect (all eight subjects sorted each $X$ condition more rapidly than the corresponding $\mathrm{W}$ condition). ${ }^{6}$ Additionally, these data appear to reflect a Stimulus Type by Color Set interaction $[F(1,7)=11.04, p<.05$; also, the difference between the mean classification times of RYB-X and RYB-W was greater than between ROY-X and ROY.W for seven of eight subjects, with one "tie"]. Bearing in mind appropriate caution about interpreting noncrossover interactions with reaction time data, this pattern is similar to that of the Set Size 3 scanning tasks in Experiment $\mathrm{I}$. The amount of verbal interference seems to be dependent upon sensory organization factors as well as the number of "items" which are grouped in a response category.

\section{Discussion}

The pattern of results in the speeded classification tasks generally confirms two principles observed in the scanning tasks. (1) "Simple" classifications which allow discrimination of a single ink color or focusing upon
Table 2

Mean Classification Time (in Seconds) Per Stimulus Card in Experiment II

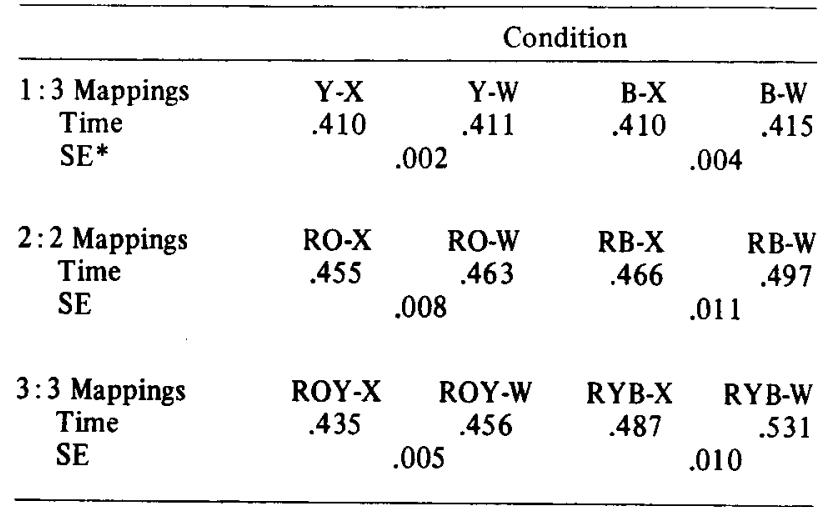

Note-Each cell entry is the mean sorting time per card for decks of 36 cards for the $1: 3$ and 2:2 mapping tasks, and 30 cards for 3:3 mapping tasks.

*Standard error of the difference for deck type (X vs. W), calculated separately for each color set.

small groupings of similar colors appear to involve rapid "visual" perceptual decisions which are not disrupted by verbal response competition. (2) "Complex" classifications requiring the grouping of nonadjacent hues not only produce longer classification times, but also such tasks are slowed even more by the presence of incongruent color names. On the whole, this pattern of agreement suggests that scanning and sorting may involve similar decision processes.

There is a large departure between our results and those of Hock and Egeth (1970), who observed substantial verbal interference when subjects were required to sort out a single ink color in a 1:4 mapping. Methodological differences between our scanning and sorting procedures are, however, numerous and probably nontrivial. Hock and Egeth required their subjects to turn up face-down cards, place them into boxes labeled "yes" or "no," and simultaneously orally respond "yes" or "no." It seems likely that the requirements of the oral response, in addition to the individual (nonoverlapping) presentation of the stimuli, may well have a bearing on the level of processing at which a perceptual decision is made. We would argue, on the other hand, that our "manual" tasks provide a closer approximation of how visual scanning is used continuously in searching a visual array in "real world" situations, and may provide a better assessment of optimal visual processing.

In addition, our data demonstrate that sensory organization factors (i.e., which particular colors are grouped together) exert an important influence on classification time and susceptibility to verbal interference within set size. It does seem inappropriate to collapse across different color groupings of the same size in order to fit slope-intercept functions (Sternberg, 1969), since what appears to constitute the "memory load" is not merely set size, but the visual encodability and discriminability of a particular set of colors as well. 


\section{GENERAL DISCUSSION}

We have demonstrated that in two perceptual tasks. scanning and speeded classification, the occurrence of Stroop-like interference from "irrelevant" words is dependent upon the specific stimulus classifications required on a given trial. Quite successful gating of the word is possible when subjects are required to discriminate only a single color or a grouping of highly similar ink colors, suggesting perceptual decisions independent of verbal processing. On the other hand, considerable verbal interference resulted when the task required the grouping of several dissimilar colors. This apparent intrusion of verbal interference raises the issues of the relative efficiency of visual and verbal codes in performing perceptual classifications and the locus of the interference.

\section{Locus of the Interference}

One possible interpretation of the Stroop-like interference effect is that, under conditions of a large memory load, subjects attempted name-level rather than image-level memory comparisons. A name code strategy may have resulted from either an inability to maintain image codes for three nonadjacent hues, or perhaps because a name search could be executed more rapidly than a visual search, given the memory require. ments of the task. In either case, a memory search for names would require at least covert naming of the color patch stimuli, and it seems likely that this naming would be disrupted by reading the incongruent word in fundamentally the same manner as incongruent printed names disrupt overt color naming in the traditional Stroop test.

An alternative interpretation is that the large visual memory load slows the decision time for each color patch sufficiently that reading of the word elicits competing color imagery, which interferes with the memory comparison process before its completion.? According to this interpretation, it is the stimulus categorization time rather than the memory load per se which determines whether interference will occur.

While the latter interpretation of the interference effects may appear more parsimonious, in that it does not postulate a shift in strategy as a function of the memory set, informal reports from several subjects suggest that they found it difficult to "keep orange, green, and purple in mind at the same time," without active verbal rehearsal of the names. Previous research with Stroop-like tasks (e.g., Dyer, 1973; Flowers, 1975) suggests that performance may be disrupted by either verbal response competition or image competition, depending on the task requirements. Since our present data offer no definitive evidence about which form of encoding was primarily involved, the exposition of a single detailed process model does not appear useful.

\section{Performance Limitations in Visual Classification}

From a broader perspective, the present results illustrate the necessity to consider the source of performance limitations in a visual classification task when predicting whether performance will be influenced by the presence of additional relevant or irrelevant stimulus information. Garner (1970) and Flowers and Garner (1971) have emphasized the importance of distinguishing tasks in which the stimuli are of low visual contrast and/or brief duration (state limited) from those tasks in which an equivalent level of difficulty is produced by visual similarity among the stimulus alternatives (process limited). Recently, Norman and Bobrow (1975) have argued that it is of equal importance to distinguish between these two types of limitations (which they refer to as data limitations) and performance limitations dependent upon the allocation of higher order processing channels (resource limitations), particularly when predicting whether or not interference effects will be noted. Our present data would appear to provide an excellent illustration of performance which is primarily data limited for some perceptual groupings (when no interference results) and primarily resource limited for others (when interference occurs). Regardless of the precise form of encoding within which the interference occurs, it can be viewed as the "sharing" of resources with the processing of information from the incongruent word. Furthermore, it is apparent from our data that the allocation of processing resources for color classification is clearly not a simple function of memory set size, but is highly dependent upon the sensory dimensional structure among the stimuli assigned to a common response category or memory set.

\section{REFERENCES}

Conen. G. Some evidence for parallel comparisons in a letter recognition task. Quanerly Journal of Experimental Psichology, 1969. 21. 272-279.

DALRYMPLE-AlFord. E. C., \& AzKoul. J. The locus of imcerterence in the Stroup and related tasks. Perception \& Psichophysics. 1972.11, 385-388.

DYFr. F. N. The Stroop phenomenon and its use in the study of pereptual cognitive, and response processes. Memony \& Cognition, 14"3. 1. 106-120.

Ecieth. H. E. Biecker. D. L., d Kamlet, A. S. Verbal inferterence in a perceptual comparison task. Perception \& Paychophsics. 1969. 6. 355-356.

Flowers. J. H. \& Garner. W. R. The effect of stimulus chement redundaney on speed of discrimination as a function of state and process limitation. Perception \& Psychophysics. 1971, 9. 158-160.

Flowers. J. H. The effect of correlated linguistic dimensions on speeded classitication of visually presented trigrams. Memon \& Cognitiom. 1974. 2. 372-378.

Flowers, J. H. "Sensory" interterence in a nord-color matching lask. Porception \& Psychophysics. 1975, 18. 37-43.

Garner. W. R. The stimulus in formation processing. Americun Psychologist. 1970). 25. 350-358.

Gottwald, R. L.. d Garner. W. R. Effects of focusing strategy on speeded classification with grouping. filtering. and condensation tasks. Perception \& Psychophysics. $1971.11,179.182$. 
Hock. H. S., \& EgETH, H. E. Verbal interference with encoding in a perceptual classitication task. Journal of Experimental Psichology. 1970. 83. 299-303.

Norman. D. A.. \& Bobrow, D. G. On data limited and resource limited processes. Cognitive Psychology, 1975 , 7, 44-64.

Pisoni. D. B.. \& TASH, J. Reaction times to comparisons within and across phonetic categories. Perception \& Psychophysics, 1974, 15, 285-290.

Posner. M. I., Boies, S. J., Eichelman, W. H., \& TAYLOR. R. L. Retention of visual and name codes of single letters. Joumal of Experimental Psychology Monograph, $1969,79,1-13$.

Posner, M. I., \& Mitchell, R. F. Chronometric analysis of classification. Psychological Review, 1967, 74, 392-409.

ReED, S. K. Psychological processes in pattern recognition. New York: Academic Press, 1973.

SternberG, S. High-speed scanning in human memory. Science, 1969, 153, 652-654.

Stroop, J. R. Studies of interference in serial verbal reactions. Journal of Experimental Psychology, 1935, 18, 643-662.

Treisman, A. M., \& Fearnley, S. The Stroop test: Selective attention to colours and words. Nature, 1969, 222, 437-439.

Tversky. B. Pictorial and verbal encoding in a short-term memory task. Perception \& Psychophysics, 1969, 6, 225-233.

Uleman, J. S., \& ReEves, J. A reversal of the Stroop effect through scanning. Perception \& Psychophysics, 1971, 9. 293-295.

Weber, R. J., \& Castleman, J. The time it takes to imagine. Perception \& Psychophysics, 1970, 8, 165.168.

\section{NOTES}

1. Each W list used within a block of trials was balanced for the number of consecutive repetitions of each ink color with the $\mathrm{X}$ list used within that block. The authors are deeply appreciative of Gina Newbold's efforts in constructing stimulus lists with these properties.

2. The incongruent words were equally distributed among the names of the other ink colors in the deck for all " $W$ " conditions used in this experiment.

3. An unexpected shortage of volunteer subjects from the introductory psychology class required our department to adopt subject rationing procedures, which limited both the number of subjects available and the time required of each subject. Since the $1: 3,2: 2$, and $3: 3$ mapping tasks were statistically treated as separate experiments in the present case, no problems are caused by the unequal subject distribution.

4. Subjects rarely looked at the sample card for more than a brief glance following the first block of trials, since there were only two different color assignments required.

5. $t(11)=.45$ for a comparison between $Y \cdot X$ and $Y-W$; $t(11)=1.30$ for a comparison between $B-X$ and $B-W$.

6. Analysis of variance reflected the effect of color set $[F(1,7)=33.8, p<.005]$, and the effect of list type $[F(1,7)=$ $35.6, \mathrm{p}<.01]$. A small Stimulus Type by Blocks of Trials interaction was noted $[F(6,42)=2.3, p<.05]$, but this appears to stem from differential room for improvement with practice.

7. We are indebted to an anonymous reviewer for suggesting this interpretation.
(Received for publication August 8, 1975; revision accepted October 27,1975 .) 